\title{
Exact Reachability Analysis for Planning Skew-Line Needle Arrangements for Automated Brachytherapy
}

\author{
Animesh Garg ${ }^{1}$, Timmy Siauw ${ }^{2}$, Guang Yang ${ }^{1}$, Sachin Patil ${ }^{3}$, \\ J. Adam M. Cunha ${ }^{2}$, I-Chow Hsu ${ }^{2}$, Jean Pouliot ${ }^{2}$, Alper Atamtürk ${ }^{1}$, Ken Goldberg ${ }^{1,3}$
}

\begin{abstract}
When planning skew-line needle arrangements for automated brachytherapy, one objective is to identify a set of candidate needles that enter from a specified entry region, avoid specified organs-at-risk and sufficiently cover the target (tumor) volume. Existing methods use uniform or random sampling to generate a set of candidate needles, which may not adequately cover the target volume. In this paper we present an exact reachability analysis that can be used to guide the selection of candidate needles and to identify which subset of the target volume may not be reachable. Assuming linear needles, convex polyhedral representations of entry zone, organs-at-risk and target volume, we give an exact polynomial time algorithm for checking existence and calculation of the non-reachable set in the target volume. We perform experiments using patient data from 18 brachytherapy cases and found that 11 cases had nonempty occluded volume inside the target ranging from $0.01 \%$ to $4.3 \%$ of target volume. We also report a sensitivity study showing the change in the occluded volume with dilation of the avoidance volume and entry zone.
\end{abstract}

\section{INTRODUCTION}

Many problems in computer graphics, robotics and automation require an exact reachability (or visibility) computation in presence of obstacles in 3D. This study explores a non-traditional application of this body of work for a cancer therapy called brachytherapy, which is a form of radiation therapy where the radioactive source is placed in proximity to the tumor. Prostate cancer has a high incidence among men, accounting for $14.4 \%$ of all cancers diagnosed and $5.1 \%$ of all cancer deaths [1], and brachytherapy is a common and successful treatment modality for prostate cancer. In prostate brachytherapy, the radioactive source is delivered to the tumor site using an arrangement of temporarily inserted needles. Currently, this needle arrangement is restricted to only parallel needles by a needle guide; often entailing punctures in healthy organs to reach the target, which in turn may lead to trauma and side effects.

It has been shown that manually created skew-line (i.e. non-parallel, non-intersecting) needle arrangements [2] can produce dose plans on or above par with standard clinical patterns. Further, optimized needle positions, even limited to

This work was supported by NSF Award IIS-1227536, CITRIS seed grant, NSF Award 0970180, National Security Science and Engineering Faculty Fellowship from the Office of US Secretary of Defense.

${ }^{1}$ Author is with IEOR University of California, Berkeley, CA, USA \{animesh.garg, gy8, atamturk\}aberkeley.edu

${ }^{2}$ Author is with Radiation Oncology, UC, San Francisco, CA, USA $\{$ SiauwK, CunhaA, ihsu, JPouliot\} @ radonc.ucsf.edu

${ }^{3}$ Author is with EECS, University of California, Berkeley, CA, USA \{sachinpatil, goldberg\}aberkeley.edu

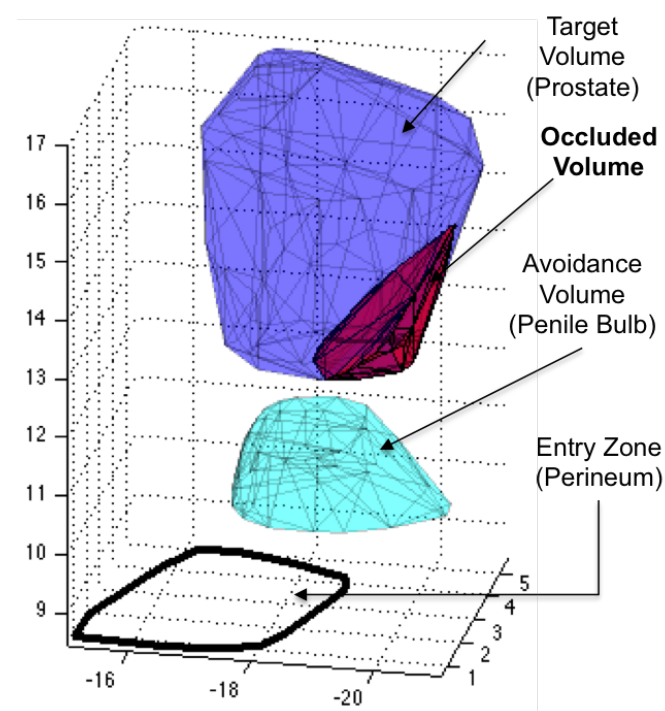

Fig. 1: This figure shows a target volume (blue), an avoidance volume (cyan), and an entry zone (black), which represent the prostate, penile bulb, and perineum, respectively, in case of prostate brachytherapy. This study develops an exact algorithm for finding an occluded volume (red), which is the region inside the target volume that cannot be reached by any straight line originating in the entry zone without intersecting the avoidance volume, or showing that no such volume exists. This algorithm could be an important component of needle planning algorithms for computing brachytherapy needle arrangements.

parallel needles, may require fewer needles to achieve a similar plan [3]. Use of optimized skew line needle arrangements can achieve above par radiation dose distribution without puncturing healthy organs [4]. Previously the authors have developed Needle Planning by Integer Program (NPIP) [5], an algorithm for planning patient-specific, skew-line needle arrangements, and explored a robotic-assisted insertion of optimized needle arrangements into phantom tissue [6].

In overview, the needle planning problem is to determine a set of non-intersecting line segments that: (a) originate in a pre-specified entry zone, (b) do not puncture any avoidance volume, and (c) provide spatial coverage of target volume. "Spatial coverage" implies that every point in target volume is within a prespecified distance $(\delta)$ of atleast one needle. Figure 1 shows a target volume, avoidance volume, and entry zone, which represent the prostate, penile bulb, and perineum, respectively, in case of prostate brachytherapy.

NPIP poses this as a set cover problem and optimizes over a large set of randomly generated candidate needles to select a minimal subset providing spatial coverage. Each can- 
didate needle originates in Entry zone, passes through Target volume without intersecting Avoidance volume. However, random generation may result in "voids" in the target volume that are not spatially covered by any candidate needle. This can lead to an inadequate dose to the target and diminish the efficacy of treatment. These voids can be a result of inadequate sampling or because the volume is "occluded", i.e. it is unreachable from the entry zone without intersecting avoidance volume.

Occluded volume calculation is a step towards systematic needle selection to guarantee absence of voids in reachable region and providing complete coverage in unreachable region. This study develops an algorithm for exact characterization of an occluded volume. We model this problem as a linear program which encodes the geometric properties of the prostate. Specifically, our contributions are:

(a) an exact algorithm for finding an occluded volume that exploits the geometric structure and constraints of prostate brachytherapy,

(b) a check for the existence of an occluded volume that is polynomial time in the number of vertices describing the relevant volumes.

We also perform a sensitivity analysis with the motivation that exact contouring of avoidance volume/entry zone is often not possible due to a combination of limitation of imaging and conservative clinical estimates of anatomy contours. And a small uncertainty can result in a significant occluded volume.

We use our algorithm to find the occluded volume, or show that none exists, for data sets from brachytherapy patients treated at the UCSF clinic. Given the uncertainty in defining anatomical structures, effect of systematic perturbations of the avoidance volume and entry zone on occluded volume are analyzed. Although this study only considers a system with only a single target, avoidance volume, and entry zone, our algorithm is extensible to multiple targets, avoidance volumes, and entry zones. Furthermore non-convex structures can be handled by decomposition into convex sub-structures and a union operation at the end.

\section{BACKGROUND AND RELATED WORK}

The problem of finding occlusions has been well-studied in computer graphics and computational geometry. Scherzer et al. [7] provide a survey in shadow computations. These are often performed at a pixel level exploiting parallel computing for handling real-time rendering of large discrete models. CGAL [8] provides efficient algorithms for such calculations. Recent work has also focused on sub-pixel shadow mapping for handling issues like jagged shadows and aliasing has been discussed in [9, 10]. Research in automation has looked at similar problems regarding polyhedral assembly[11] and view point selection [12].

This study examines the problem of checking existence of occluded volume in a polyhedral complex. Our algorithm exploits assumptions about the geometry of prostate brachytherapy, which we use to simplify the problem statement compared to a general occlusion calculation problem.

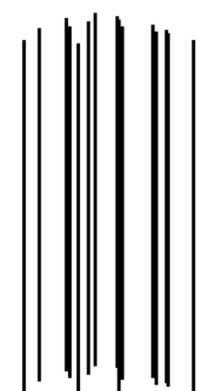

(a)

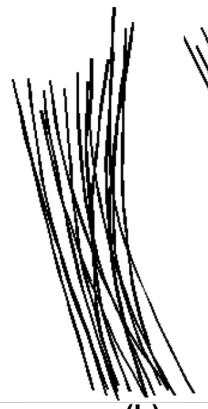

(b)

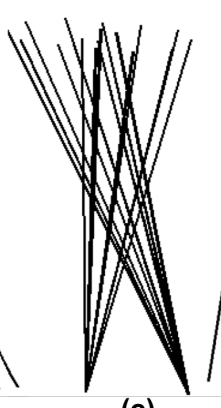

(c)

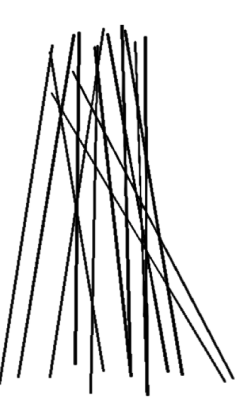

(d)
Fig. 2: The figure shows the progression in body of work in needle configurations in prostate Brachytherapy. Clinical practitioners regularly use needle templates with parallel needles as in (a) [18], which were improved upon by the freehand technique in (b) [19]. Fireworks needle configurations as in (c) were proposed with skew needles to avoid puncture in healthy organs [14]. And latest needle planning system coupled with automated needle insertion allows use of any point in the entry zone as in (d) [5].

We develop a linear program that is feasible if and only if an occluded volume exists. Thus the existence of the occluded volume can be checked in polynomial time in the total number of vertices (i.e., of the target volume, avoidance volume, and entry zone) without having to perform expensive polyhedron intersection calculations.

Our goal is to introduce automation into the brachytheratpy treatment process. We have worked on several aspects of the problem: treatment planning [4, 13], needle configuration planning [5, 14], robotic needle placement [6, 15], and recently the use of custom guides for brachytherapy needle placement [16, 17].

In prostate brachytherapy, an arrangement of hollow needles is inserted into the prostate through the perineum, the patch of skin between the testicles and anus. Radiation dose is delivered to the prostate by sequentially threading a radioactive source through each needle. The dose distribution is controlled by halting the source at pre-specified locations along each needle for some dwell time. Most clinicians insert needles using a rigid template with parallel holes as a needle guide [18]. However, this template restricts the possible insertion locations and directions, which can make puncturing obstructions such as penile bulb unavoidable. Puncturing healthy tissues results in trauma related sideeffects such as tissue swelling; urinary infections \& incontinence; and impotence [20, part 7], [21]. A freehand approach for needle insertion based on trans-rectal ultrasound guidance was developed [19]. This technique gives the physician some flexibility to avoid puncturing healthy tissue, but it requires experience to master.

Siauw et al. [5] recently proposed a novel algorithm, NPIP, which computes patient-specific needle arrangements based on skew line segments. Since these needles can be inserted at a continuum of angles, it is non-intuitive for a human operator to implant the configuration. However, such a skew line needle arrangement can be implanted using an automated needle insertion robot as in Garg et al. [6, 22] or a custom needle guide. The development of needle planning is shown 




(a)

(b)
Intersection of Only Truncated

cclusion Cones From Extreme Points Volume (0)

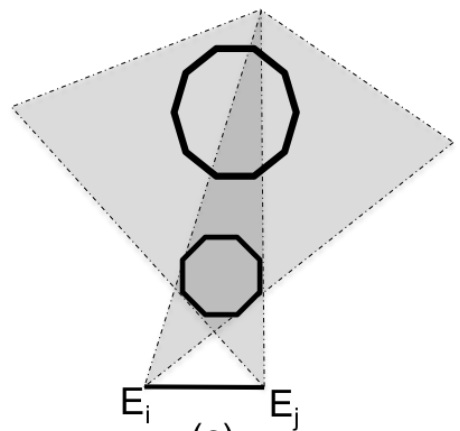

(c)

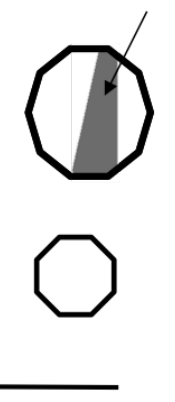

(d)

Fig. 3: The figure illustrates the proposed algorithm for calculation of Occluded volume $\mathcal{O}$. (a) We begin with input geometry with three volumes of interest: Target $(\mathcal{T})$, Avoidance $(\mathcal{A})$ and Entry Zone $(\mathcal{E})$. Fig (b) shows possible occlusion cones $C_{e}, \forall e \in E$ from different locations at entry zone. (c) However only cones generated from extreme points $\left(C_{E_{i}}\right)$ of the entry region need to considered to calculate occluded volume. Lastly as in (d) The polyhedral representation of $\mathcal{O}$ is output as the intersection of $\mathcal{T} \cap\left(\cap C_{E_{i}}\right)$

\section{in Figure 2.}

Dose planning has three main steps - candidate needle generation, needle selection, and dose planning. The candidate needle set is generated by randomly sampling line segments that originate from the entry zone are inside a projection of the prostate. The needle selection step finds a subset of candidate needle, such that every point in the target is within a distance parameter $(\delta)$ from atleast one needle in the subset. The heuristic helps ensure that the target can be covered with adequate dose during dose planning. Dose planning is done using Inverse Planning by Integer Program (IPIP) [4], which maximizes dose coverage of the target using only the needles in the subset within dose limits to healthy tissue.

Often there are points in the target volume that cannot be covered by any needle in the candidate needle set (i.e. they are not within the user-specified distance from any candidate needle). Currently, these points are ignored in the needle selection step. This simplification does not significantly impact the final solution if the number of ignored points is small. However, for a given geometric configuration and a parameter $\delta$, it is desirable to provide a guarantee of complete coverage of the target or a proof that no needle arrangement can achieve complete coverage.

The algorithm presented in this study for calculation of an exact occluded volume, is a step towards this coverage guarantee. Such a guarantee could provide bounds on treatment quality. Our approach would result in a complete algorithm as opposed to the heuristic approach from NPIP. Using the occluded volume, an informed selection of needles can be made for subsequent dose planning. For instance, systematic placement of needles around the occluded volume can achieve desired dose coverage despite incomplete spatial coverage and unreachability of the occluded volume.

\section{Problem Statement}

Notation: Given a target volume $\mathcal{T}$, an avoidance volume $\mathcal{A}$, and an entry zone $\mathcal{E}$, we are interested in finding an analytic description of an "occluded volume" $\mathcal{O}$, contained within the target volume $\mathcal{T}$ or show that no such "occluded volume" $\mathcal{O}$ exists. The latter case implies that every point in the target volume $\mathcal{T}$ can be reached by a line segment from some point in the entry zone $\mathcal{E}$ without intersecting the avoidance volume $\mathcal{A}$.

In the context of brachytherapy, absence of an occluded volume implies that at least one needle can reach every point inside the target (prostate) from the pre-specified entry zone. Assumptions: We have assumed that the objects in the environment can be represented as finite polyhedra, and hence are convex. In case of brachytherapy, the organs are contoured by a physician and polyhedral representation results in the convex hull of the contour. We work with a planar region $\mathcal{E}$, represented by its extreme points. Furthermore, there is no intersection between either of the objects: $\mathcal{T}, \mathcal{A}$ and $\mathcal{E}$.

Stated formally, we are interested in finding $\mathcal{O} \subseteq \mathcal{T}$, such that for each $p \in \mathcal{O}$, there exists a $\lambda \in[0,1]$ such that: $[\lambda e+(1-\lambda) p] \in \mathcal{A}$, for all $e \in \mathcal{E}$, or show that no such region $\mathcal{O}$ exists for the given configuration of $\mathcal{T}, \mathcal{A}$ and $\mathcal{E}$.

Every polyhedral object in the environment can be specified completely by the vertex set of its convex hull. Let a convex polyhedral avoidance volume $\mathcal{A}$ be represented by the vertex set $A$ of its convex hull. The sets $T$ and $E$ are defined similarly using $\mathcal{T}$ and $\mathcal{E}$. Further, defining $J:=\left\{j: A_{j} \in A\right\}$ as the index set of points in $A$. The index sets $K:=\left\{k: T_{k} \in T\right\}$ and $I:=\left\{i: E_{i} \in E\right\}$ are defined similarly for $T$ and $E$, respectively.

Definition: A truncated occlusion cone $C_{e}$ is the polyhedron which characterizes the occluded volume generated by a convex set of vertices $A$ as viewed from a point $e \notin$ $\operatorname{Conv}(\mathcal{A})$. As shown in Figure 3, $C_{e}$ consists of the faces of $\mathcal{A}$ visible from $e$ and all points behind them generated as a conic hull of rays corresponding to visible extreme points. Formally,

$$
\begin{gathered}
C_{e}=\left\{x \in \mathbb{R}^{n}: x=e+\sum_{j \in J} \lambda_{j}\left(A_{j}-e\right),\right. \\
\left.\sum_{j \in J} \lambda_{j} \geq 1, \quad \lambda_{j} \geq 0, \forall j \in J\right\}
\end{gathered}
$$


In case of a non-planar specification of $\mathcal{E}$ we can find a suitable projection on a plane. In case of non-convex objects, we can represent them as a disjoint union of convex sets. The algorithm outputs an occluded volume for each convex subset, and the union of these results in final occluded volume. We note that in this case the final occlusion region may not be necessarily convex.

\section{Algorithm}

The proposed algorithm exploits the observation that every point $p$ in the intersection of truncated occlusion cones $C_{i}$ generated from vertices $E_{i}$ of a convex closed region $\mathcal{E}$ also lies in the truncated occlusion cone $C_{e}$ generated from any other point $e$ in region $\mathcal{E}$. Stating the above formally:

$$
\begin{aligned}
& \forall p \in \bigcap_{i \in I} C_{E_{i}}, \exists \bar{\lambda} \in \mathbb{R}^{A}: \sum_{j \in J} \bar{\lambda}_{j} \geq 1 ; \bar{\lambda} \geq 0 \\
& \text { such that: } p=e+\sum_{j \in J} \bar{\lambda}_{j}\left(A_{j}-e\right) \text { i.e. } \quad p \in C_{e}, \\
& \text { where } e=\sum_{i \in I} \mu_{i} E_{i}, \sum_{i \in I} \mu_{i}=1, \mu_{i} \geq 0, \forall i \in I
\end{aligned}
$$

This entails that any point in $\mathcal{T}$ occluded from $E_{i}, \forall i \in I$ is also occluded from any point in $\mathcal{E}$. Every point $p \notin \bigcap C_{i}$ is classified as visible, since $p \in C_{i}$ for at least one $i \in I$. Also, every point $p \in C_{e}$ is either visible from at least one $E_{i}$, i.e. $p \notin C_{i}$, or lies in the occluded volume $\mathcal{O}$, if it exists, i.e. $p \in \bigcap C_{i}$.

An intuitive explanation for this observation is if a point $p \in \bigcap C_{i}$, then none of the $E_{i}$ 's can see $p$. Moreover, by reversibility of visibility, $p$ can't see any of the $E_{i}$ 's either. Since $\mathcal{E}$ is convex, hence by extension $p$ should not be able to see any other point in $\mathcal{E}$. Moving the viewing perspective to any point $e \in \mathcal{E}$, and by similar argument as above, any $e \in \mathcal{E}$ can't see $p$ either.

This observation significantly improves the computational effort in calculation of the occluded volume, since we only need to generate truncated occlusion cones on the finitely many vertices of the convex region $E$, instead of every point in $E$, or a discretization thereof.

Hereafter, we build a linear system system of equations to check for existence of an occluded region $\mathcal{O}$. The system 3 is feasible if $\mathcal{O}$ is non-empty and infeasible otherwise. If the system of equations return feasibility, we calculate the polyhedral representation of $\mathcal{O}$ using an iterative method for intersecting polyhedrons.

\section{A. Check for Existence of Occlusion Volume $\mathcal{O}$}

Assume $\mathcal{O}$ is non-empty, and let a point $x \in \mathcal{O}$. Then by definition, $x$ must have the following two properties:

(1) It must be a convex combination of the vertices of $\mathcal{T}$ (since $\mathcal{O} \subseteq \mathcal{T}$ ).

(2) The line segment between $x$ and some point $e \in \mathcal{E}$ must have atleast one point that is a linear combination of the vertices of $\mathcal{A}$.

$$
\begin{aligned}
x= & \sum_{k \in K} \mu_{k} T_{k}, \\
& \sum_{k \in K} \mu_{k}=1, \quad \mu_{i} \geq 0, \forall i \in I,
\end{aligned}
$$

which is simply the definition of convex combination representing the first condition.

While the second condition is represented by $x \in C_{e}$. In other words, the conic hull of $A$ from $e$ is the set of rays originating at $e$ that have at least one point in $A$, i.e., at least one point that is a linear combination of the vertices of $\mathcal{A}$. So by definition, $x$ must be in the intersection of $C_{e}, \forall e \in \mathcal{E}$.

$$
x \in \bigcap_{e \in \mathcal{E}} C_{e}
$$

To simplify our representation of this property of $x$, which requires an intersection of an infinite number of conic hulls, it is sufficient to find the intersection of the conic hulls of $A$ around $E_{i}, i \in|E|$, the vertices of $\mathcal{E}$. That is,

$$
\bigcap_{e \in \mathcal{E}} C_{e}=\bigcap_{i \in I} C_{E_{i}}
$$

We can then represent the second property of $x$ using

$$
\begin{gathered}
x=E_{i}+\sum_{j \in J} \lambda_{i j}\left(A_{j}-E_{i}\right), \quad \forall i \in I \\
\sum_{j \in J} \lambda_{i j} \geq 1, \quad \forall i \in I, \lambda \geq 0,
\end{gathered}
$$

which states that $x$ must be in the conic hull of $A$ originating at each $E_{i}, i \in I$. We note that the condition: $\sum_{j} \lambda_{i j} \geq$ $1, \forall i \in I$, enforces that only the points situated beyond the visible boundary of the avoidance region $\mathcal{A}$ are included.

Put together, we get our linear system, (LS).

$$
\begin{aligned}
& \text { (LS) } x=E_{i}+\sum_{j \in J} \lambda_{i j}\left(A_{j}-E_{i}\right), \forall i \in I \\
& x=\sum_{k \in K} \mu_{k} T_{k}, \\
& \sum_{k \in K} \mu_{k}=1, \mu \geq 0 \\
& \sum_{j \in J} \lambda_{i j} \geq 1, \forall i \in I, \lambda_{i j} \geq 0, \forall i \in I, \forall j \in J .
\end{aligned}
$$

By construction, any $x$ value that satisfies (LS) cannot be connected to a point in $\mathcal{E}$ by a line segment that does not intersect $\mathcal{A}$. Therefore, the feasible region of $x$ in (LS) is exactly $\mathcal{O}$, the occluded volume. Consequently, if (LS) is infeasible, then $\mathcal{O}$ is empty and the entire target volume can be reached by at least one line segment originating in the entry zone that does not intersect the avoidance volume. If (LS) is feasible, then $\mathcal{O}$ is not empty, and it is worth noting that since the feasible region is made up from a set of linear constraints and is bounded by $\mathcal{T}$, that $\mathcal{O}$ is a polytope when it is not empty. 




\section{B. Calculation of Occlusion Volume $\mathcal{O}$}

We use algorithm 1 to obtain the polytope $\mathcal{O}$. Polytopes can be described using inequalities (H-polytopes) or vertices (V-polytopes). H-polytopes can be converted to V-polytopes using vertex enumeration and vice-versa by facet enumeration. In this case, input is in the form of V-polytopes while output as H-polytope for a complete representation of $\mathcal{O}$. A preliminary approach for calculation of intersection of convex polyhedra is to start by converting the input polyhedra to H-representation. Then, the intersection is a redundancy removal problem in the union of inequality systems. The minimal H-representation of the union results in H-polytope $\mathcal{O}$. Furthermore, to get vertices of $\mathcal{O}$, we can solve a vertex enumeration problem.

\section{Complexity Analysis}

The linear system of equations for checking existence of a occluded volume can be solved as an LP. Assuming $A$, $T$ and $E$ have $m_{1}, m_{2}$ and $m_{3}$ points respectively and dimension of space being $n$. Furthermore, also assuming nondegeneracy we have: $n \leq m_{1}, m_{2}, m_{3}$. Then, the system 3 has $\left(n+m_{1}+m_{2} m_{3}\right)$ variables and $\left(n\left(m_{2}+1\right)+m_{3}+1\right)$ constraints apart from the non-negativity constraints. LP has known polynomial time complexity [23].

Furthermore, in case there exists an occluded volume or the linear system 3 returns feasible solution, we calculate truncated occlusion cones $C_{E_{i}}, \forall i \in I$. Thereafter, a sequence of convex polyhedron intersections are performed to obtain $C:=\bigcap C_{E_{i}}$. Every occlusion cone $C_{e}$ has $O\left(m_{2}\right)$ extreme rays. We perform $m_{3}$ such intersection operations for calculating $C:=\bigcap C_{E_{i}}$, and thereafter one more intersection with target volume $T$ to get obtain $O$. Hence the complexity for the intersections is in $O\left(m_{2} m_{3}\right)$. Polyhedral intersection in general is shown to be NP-Hard [24]. However, using the above approach, solving for redundancy in union of $\mathrm{k} \mathrm{H}$ polytopes is an LP for convex polyhedra. An intersection of two such occlusion cones can be performed in polynomial time for low dimension [25].

\section{EXPERIMENTS AND RESULTS}

\section{A. Preprocessing Clinical Dataset}

We tested our algorithm on 18 anonymized anatomy cases taken from patients previously treated at UCSF. This data set contained surface points on the prostate and the penile bulb, which were termed as the target volume $\mathcal{T}$ )and avoidance volume $\mathcal{A}$, respectively. The prostate volumes ranged from 27 to $97 \mathrm{~cm}^{3}$ and the penile bulb volumes ranged from 1 to $11 \mathrm{~cm}^{3}$.

A specification of the entry zone did not exist in the data set because segmenting an entry zone is not a standard practice in HDR-brachytherapy for prostate. In clinical procedures, the available entry zone near the perineum is visually assessed by the physician during needle insertion. We used the needles entry points in the actual cases as the available region. For this study, the entry zone was defined as the convex hull of these needle entry locations projected on a plane parallel to the ground ( $\mathrm{x}-\mathrm{y}$ plane) and located $2 \mathrm{~cm}$ below the penile bulb. This is a reasonable anatomical proxy for the perineum since the distance between the penile bulb and the perineum in human anatomy is also $\sim 2 \mathrm{~cm}$. This is a conservative estimate of the possible entry zone; however, it ensures that we restrict the entry zone to an area used by the physician. We note that the entry zone to allow for skew-line needles could infact be larger than this, which would reduce occlusion. The entry zone area ranged from 7 to $13 \mathrm{~cm}^{2}$.

\section{B. Occluded Volume Analysis}

We implemented our algorithm in Matlab. We used the the Matlab interface for Multi-Parametric Toolbox 3 (MPT) [26] to calculate extreme points of a convex bounded polyhedra and to perform polyhedral intersection operation. MPT toolbox implements polyhedral intersection queries in low dimension using the approach described in section IV-B. The algorithm was run on a computer with OS/X $10.9 .2 \mathrm{~m}$ $2.7 \mathrm{GHz}$ Intel core i7, and $16 \mathrm{~GB}$ memory.

Table II lists the size of occluded volumes for the different cases in the dataset. For all cases, we checked for the existence of an occluded volume in the prostate where needles cannot reach without puncturing the penile bulb. We found that 11 out of 18 patients had a non-empty occluded volume. Among the patients with occlusion, the size of the occluded volume ranged from $0.06 \mathrm{~cm}^{3}$ to $2.4 \mathrm{~cm}^{3}$ or $0.01 \%$ to $4.3 \%$ of the respective target volume. The running time ranged from $4 \mathrm{~s}$ to $9 \mathrm{~s}$. It is worth noting that time on the order of seconds is inconsequential to the overall brachytherapy work-flow.

\section{Sensitivity Analysis}

The size of the penile bulb has substantial uncertainty in its definition owing to difficultly in its identification on a CT scan. We used our algorithm to compute the occluded volume in each patient's target volume for various dilations of the nominal penile bulb. The dilations were computed by expanding the vertices of the penile bulb about its centroid by a dilation factor $(\alpha)$ that ranged from 0.75 to 1.25 in increments of 0.05 , where $\alpha=1$ results in no change (i.e. its original size). For each iteration, we recorded the size of the occluded volume, the volume of the dilated bulb, and the running time of the algorithm. 


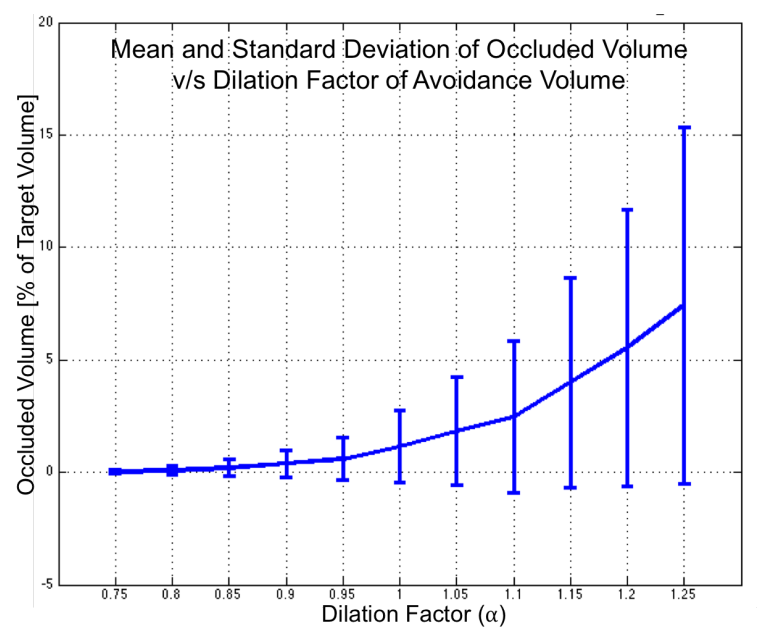

(a)

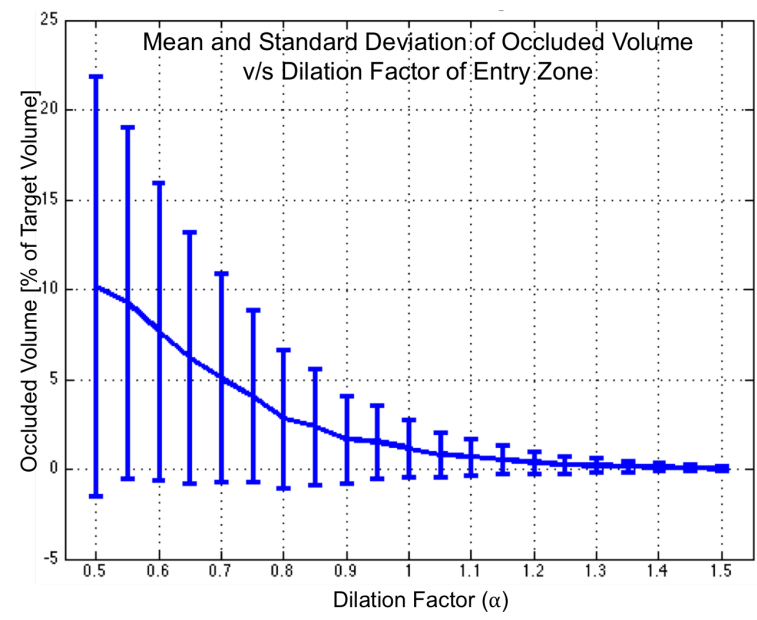

(b)

Fig. 4: The figure illustrates the change in normalized occluded volume v/s change in dilation factor $(\alpha)$ for (a) avoidance volume and (b) Entry Zone. The error bars show the standard deviation across the patients. An occluded volume value $<0 \%$ implies no occlusion exists. The graphs illustrate a possibility of large changes in occluded volume with small uncertainty in specification of either avoidance volume or entry zone.

\begin{tabular}{|r||r|r|r|r|}
\hline Px & $T\left(\mathrm{~cm}^{3}\right)$ & $A\left[\mathrm{~cm}^{3}(\% \mathrm{~T})\right]$ & $O\left[\mathrm{~cm}^{3}(\% \mathrm{~T})\right]$ & Time $(\mathrm{s})$ \\
\hline \hline 1 & 33 & $8(23)$ & $1.00(3.10)$ & 8 \\
\hline 2 & 27 & $5(19)$ & $0.00(0.00)$ & 7 \\
\hline 3 & 44 & $10(23)$ & $1.80(4.10)$ & 7 \\
\hline 4 & 31 & $4(14)$ & $0.26(0.86)$ & 5 \\
\hline 5 & 39 & $3(8)$ & $1.00(2.60)$ & 4 \\
\hline 6 & 55 & $7(14)$ & $2.40(4.30)$ & 9 \\
\hline 7 & 58 & $3(5)$ & $0.00(0.00)$ & 4 \\
\hline 8 & 38 & $4(11)$ & $0.00(0.00)$ & 6 \\
\hline 9 & 31 & $10(34)$ & $0.00(0.00)$ & 8 \\
\hline 10 & 46 & $7(15)$ & $0.06(0.12)$ & 6 \\
\hline 11 & 74 & $4(6)$ & $0.00(0.00)$ & 5 \\
\hline 12 & 32 & $4(11)$ & $0.15(0.48)$ & 5 \\
\hline 13 & 97 & $1(1)$ & $0.00(0.00)$ & 3 \\
\hline 14 & 66 & $10(15)$ & $2.30(3.50)$ & 7 \\
\hline 15 & 40 & $4(10)$ & $0.00(0.00)$ & 4 \\
\hline 16 & 50 & $6(12)$ & $0.09(0.18)$ & 5 \\
\hline 17 & 45 & $8(17)$ & $0.85(1.90)$ & 9 \\
\hline 18 & 27 & $9(34)$ & $0.003(0.01)$ & 9 \\
\hline
\end{tabular}

TABLE I: Results from 18 Patient Cases listing Target volume $T$, Avoidance Region Volume $A$, Occluded Volume $O$ in absolute and as percentage of Target volume. The last column lists time required for computation in seconds.

Likewise, entry zone specification used in the dataset is a conservative estimate. Dilation of the entry zone is prone to introduction (or removal) of occlusion. We have performed calculations of occluded volumes for various dilations of the entry zone about its centroid by a dilation factor $(\alpha)$ in $[0.5,1.5]$ in increments of 0.05 .

Figures $4 \mathrm{a}$ and $4 \mathrm{~b}$ show variation in existence and size of occluded volume as a \% of target volume for various values of dilation factor $\alpha$ in case of penile bulb and entry zone, respectively. The error bars for each $\alpha$ value represent the measure of the standard deviation for the whole data set. An error bar corresponding to an occluded volume $<0 \%$ implies no occlusion exists. Figure 5a shows change in occluded volume with avoidance volume (penile bulb) dilation in one particular anatomical case. Similarly, Figure $5 \mathrm{~b}$ shows the change in occluded volume with entry zone dilation for another case in the data set.

\section{DisCUSSION AND FUtURE WORK}

The occluded volume in the target, where needles starting in the entry region cannot reach without puncturing the avoidance volume (penile bulb), if it exists, is found for all 18 cases in the data set. Computational results listed in table $\mathrm{I}$ reveal that $\sim 60 \%$ (11 out of 18 ) of cases in the data set have non-zero occluded volume. Owing to the variance in location and size of the organs and the entry zone, the results ranged from no occluded volume to (4.3\%) of the prostate being occluded.

In practice, an occluded volume inside the target volume could lead to voids in needle coverage, which results in areas that are difficult to cover with adequate dose and thus make the overall treatment of the patient less effective.

Furthermore, the effect of dilation on the avoidance volume and entry zone to occluded volume emphasizes the importance the proposed approach. As noted earlier, identification of true boundaries of the penile bulb may be difficult, and hence a conservative estimate may be made. We note from Figure 4a that the occluded volume is fairly sensitive to dilation of avoidance volume. Conservative errors of $5 \%$ in specification of margins of avoidance volume may result in $>5 \%$ of the target volume being occluded in some cases.

Moreover, the entry zone is not always precisely defined. A similar analysis shows that the occluded volume is relatively less sensitive to entry zone perturbation than to avoidance volume perturbation. As noted in Figure $4 \mathrm{~b}$, a 10\% contraction in entry zone results in $\sim 5 \%$ occlusion in target volume. While on the other hand, at $40 \%$ expansion of entry zone, all cases result in 0 occluded volume.

Figures $5 \mathrm{a}$ and $5 \mathrm{~b}$ provide qualitative review of occlusion events and resulting occluded volumes for a particular case highlighting the rapid growth of occluded volume with dilation of avoidance volume and entry zone respectively.

The errors in estimating avoidance volume and entry zone may not always be isotropic as the centroidal dilation in the 

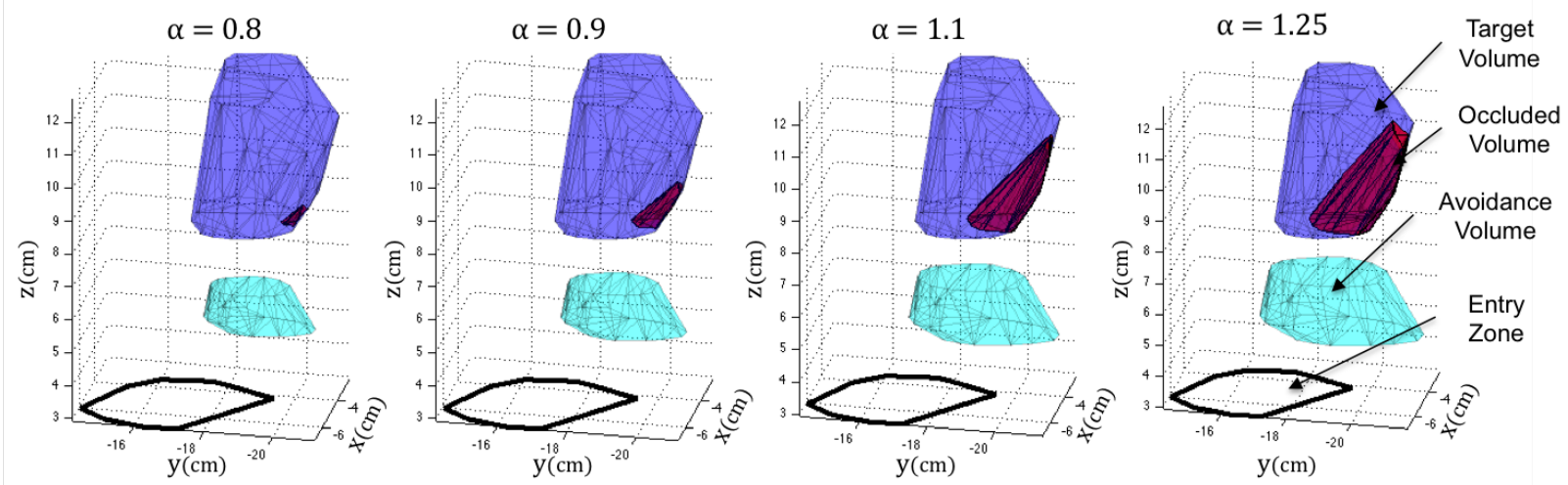

(a) Occluded volume grows with the avoidance volume.
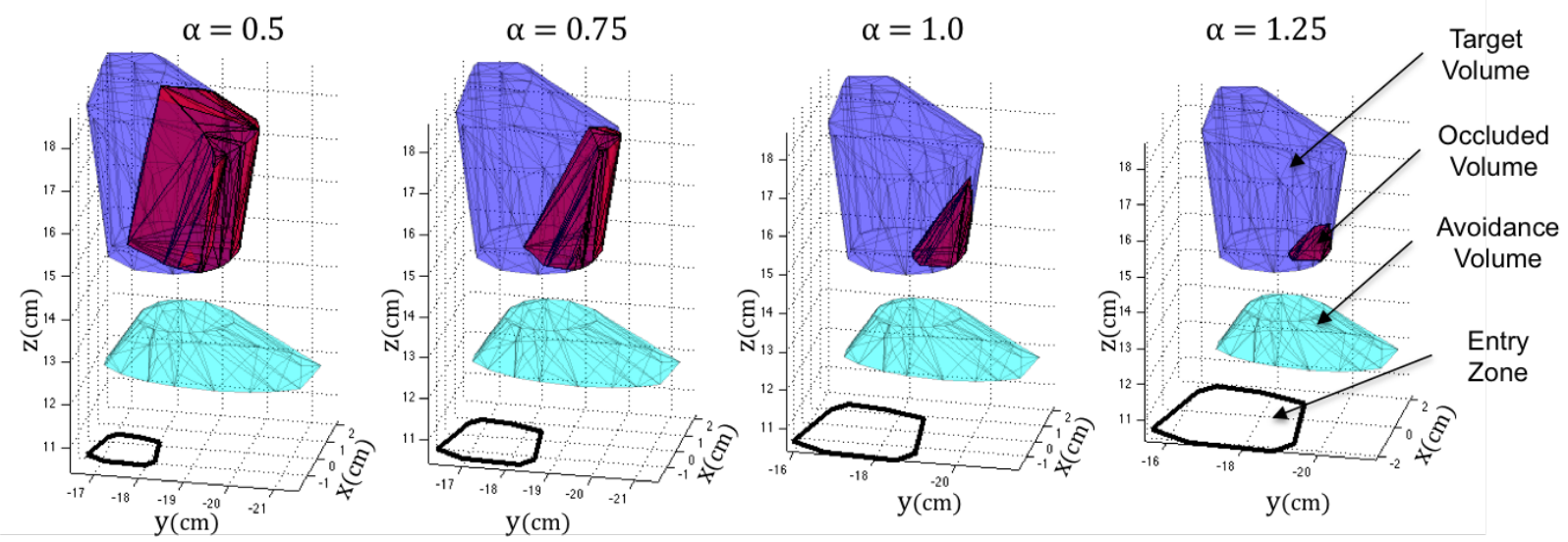

(b) As the entry zone shrinks, the occluded volume grows. In this instance, non-zero occlusion exists at even at $\alpha=1.25$.

Fig. 5: Sensitivity analysis on the occluded volume calculation yields insight on the occluded volume. Uncertainty in specification of either entry zone or avoidance volume can result in large changes in occluded volumes. The figure shows variation in occluded volume with dilation (by factor $\alpha$ ) in the avoidance volume and entry zone in two anatomy instances.

sensitivity analysis. We observe that occlusion is often resultant of obstruction from a small subset of neighboring faces. However, prior knowledge of this obstructing neighborhood is not available, necessitating a check in all directions.

Moreover, in case of non-zero occluded volume, we could perform a similar perturbation in the entry region vertices cyclically instead of isotropic dilation. This would result in identification of directions, expansion in which would result in elimination of occlusion.

Future Work: In addition to checking feasibility of a brachytherapy procedure without puncturing healthy organs, the output of the algorithm can be used to improve treatment planning. In case the target is not completely visible, we can calculate the extreme points of the entry zone which can reach the boundary of the occluded volume in target. Needles starting from these points can be adaptively reweighted for higher "importance" in the needle subset selection optimization of the NPIP algorithm [5].

Limitations: The complexity of the algorithm is proportional to number of vertex points in the convex hull of the polyhedra. A high fidelity representation would thus result in slow computations. Furthermore, for time critical applications, faster implementation of polyhedral intersection operation with use of parallel computing may be explored. Extensions:
The framework can be generalized to have more than one avoidance volumes, for instance pubic arch in the case of prostate brachytherapy. In case of a non-convex avoidance volume, a pre-processing step would section it into disjoint convex subsets. The procedure can then be used in parallel for each subset, and performing a union operation in the end.

Moreover, the algorithm is also applicable to other applications with reachability calculations: such as visual inspection, robotic spray painting, and other clinical procedures such as biopsy. Kidney biopsy is a one such problem where the physician is interested in reaching a particular region inside kidney without puncturing healthy organs.

\section{CONCLUSION}

This paper considers the problem of exact reachability analysis for checking existence of occluded volume in a target in presence of an avoidance volume not reachable from an entry zone, where all three regions of interest are modeled as convex polyhedra. The proposed algorithm performs the check in polynomial time and if it exists returns the polyhedral representation of the occluded volume. This study forms a basis for identification of candidate needle set generation and needle subset selection for treatment radiotherapy treatment planning in brachytherapy. We have shown computational results on actual anatomical cases of prostate 
cancer, and quantified the occluded volume in each case. We have found that 11 out of 18 cases have non-empty occluded volume ranging from $0.01 \%$ to $4.3 \%$ of target volume. Such occlusions can lead voids in radiation coverage, thus reducing the effectiveness of overall treatment. Furthermore, we have conducted a sensitivity analysis to study change in occluded volume with dilation of the the avoidance volume and the entry zone to emphasize that small uncertainty in specification of of regions of interest may result in large changes in occluded volumes in the target.

\section{ACKNOWLEDGEMENT}

This work has been supported in part by a seed grant from the UC Berkeley Center for Information Technology in the Interest of Society (CITRIS) and by the U.S. National Science Foundation under Award IIS-1227536: Multilateral Manipulation by Human-Robot Collaborative Systems, and by unrestricted grants from Google and Cisco. Alper Atamtürk is supported, in part, by a National Security Science and Engineering Faculty Fellowship from the Office of US Secretary of Defense and the US National Science Foundation grant 0970180. We thank our colleagues Jeff Mahler, Sanjay Krishnan and Zoe McCarthy for the feedback and suggestions.

\section{REFERENCES}

[1] N. Howlader, A. Noone, M. Krapcho et al., "Seer cancer statistics review, 1975-2010.[based on the november 2012 seer data submission, posted to the seer website, april 2013]," Bethesda, MD: National Cancer Institute, 2013, seer.cancer. gov/statfacts/html/prost.html

[2] J. Cunha, I. Hsu, and J. Pouliot, "Dosimetric equivalence of nonstandard HDR brachytherapy catheter patterns," Medical Physics, vol. 36, p. 233, 2009.

[3] E. Poulin, C.-A. C. Fekete, M. Létourneau, A. Fenster, J. Pouliot, and L. Beaulieu, "Adaptation of the cvt algorithm for catheter optimization in high dose rate brachytherapy," Medical physics, vol. 40, no. 11, p. 111724, 2013.

[4] T. Siauw, A. Cunha, A. Atamtürk, I.-C. Hsu, J. Pouliot, and K. Goldberg, "IPIP: a new approach to inverse planning for hdr brachytherapy by directly optimizing dosimetric indices," Medical Physics, vol. 38, no. 7, pp. 4045-4051, 2011.

[5] T. Siauw, A. Cunha, D. Berenson, A. Atamtürk, I. Hsu, K. Goldberg, and J. Pouliot, "NPIP: a skew line needle configuration optimization system for hdr brachytherapy," Medical physics, vol. 39, no. 7, p. 4339, 2012.

[6] A. Garg, T. Siauw, D. Berenson, J. Cunha, I.-C. Hsu, J. Pouliot, D. Stoianovici, and K. Goldberg, "Robot-guided open-loop insertion of skew-line needle arrangements for high dose rate brachytherapy," Automation Science and Engineering, IEEE Tran on, vol. 10, no. 4, pp. 948-956, Oct 2013, http://dx.doi.org/10.1109/TASE.2013.2276940

[7] D. Scherzer, M. Wimmer, and W. Purgathofer, "A survey of real-time hard shadow mapping methods," in Computer Graphics Forum, vol. 30, no. 1. Wiley Online Library, 2011, pp. 169-186.

[8] "CGAL, Computational Geometry Algorithms Library," http: //www.cgal.org

[9] E. Sintorn, O. Olsson, and U. Assarsson, "An efficient aliasfree shadow algorithm for opaque and transparent objects using per-triangle shadow volumes," in Proceedings of the 2011 SIGGRAPH Asia Conference, ser. SA '11. ACM, 2011.

[10] P. Lecocq, P. Gautron, J.-E. Marvie, and G. Sourimant, "Subpixel shadow mapping," in ACM SIGGRAPH 2013 Talks. ACM, 2013, p. 19.
[11] E. Fogel and D. Halperin, "Polyhedral assembly partitioning with infinite translations or the importance of being exact," Automation Science and Engineering, IEEE Transactions on, vol. 10, no. 2, pp. 227-241, April 2013.

[12] J. L. Alarcon-Herrera, X. Chen, and X. Zhang, "Viewpoint selection for vision systems in industrial inspection," in IEEE Int'l Conference on Robotics and Automation (ICRA), 2014.

[13] R. Alterovitz, E. Lessard, J. Pouliot, I. Hsu, J. OBrien, and K. Goldberg, "Optimization of HDR brachytherapy dose distributions using linear programming with penalty costs," Medical physics, vol. 33, p. 4012, 2006.

[14] J. Xu, V. Duindam, R. Alterovitz, J. Pouliot, J. Cunha, I.C. Hsu, and K. Goldberg, "Planning fireworks trajectories for steerable medical needles to reduce patient trauma," in IEEE/RSJ International Conference on Intelligent Robots and Systems, 2009. (IROS), 2009, pp. 4517-4522.

[15] K. Reed, A. Majewicz, V. Kallem, R. Alterovitz, K. Goldberg, N. Cowan, and A. Okamura, "Robot-assisted needle steering," Robotics Automation Magazine, IEEE, vol. 18, pp. 35-46, 2011.

[16] A. Garg, S. Patil, T. Siauw, J. Cunha, I.-C. Hsu, P. Abbeel, J. Pouliot, and K. Goldberg, "An algorithm for computing customized $3 \mathrm{~d}$ printed implants with curvature constrained channels for enhancing intracavitary brachytherapy radiation delivery," in Automation Science and Engineering (CASE), IEEE International Conference on, Aug 2013, pp. 466-473, http://dx.doi.org/10.1109/CoASE.2013.6654002

[17] S. Patil, J. Pan, P. Abbeel, and K. Goldberg, "Planning curvature and torsion constrained ribbons in $3 \mathrm{~d}$ with application to intracavitary brachytherapy," 2014.

[18] C. Salembier and P. Hoskin, "Prostate brachytherapy: High dose rate," in Prostate Cancer: A Comprehensive Perspective, A. Tewari, Ed. Springer London, 2013, pp. 739-748, http: //dx.doi.org/10.1007/978-1-4471-2864-9_62

[19] Y. Kim, I. Hsu, and J. Pouliot, "Measurement of craniocaudal catheter displacement between fractions in computed tomography-based high dose rate brachytherapy of prostate cancer," J Appl Clin Med Phys, vol. 8, no. 4, 2007.

[20] A. Tewari, Prostate Cancer: A Comprehensive Perspective: A Comprehensive Perspective. Springer, 2013.

[21] A. G. Macdonald, M. Keyes, A. Kruk, G. Duncan, V. Moravan, and W. J. Morris, "Predictive factors for erectile dysfunction in men with prostate cancer after brachytherapy: Is dose to the penile bulb important?" International Journal of Radiation Oncology* Biology* Physics, vol. 63, no. 1, pp. 155-163, 2005.

[22] A. Garg, T. Siauw, D. Berenson, A. Cunha, I.-C. Hsu, J. Pouliot, D. Stoianovici, and K. Goldberg, "Initial experiments toward automated robotic implantation of skewline needle arrangements for HDR brachytherapy," in IEEE Conference on Automation Science and Engineering, 2012, aug. 2012, pp. $26-33$, http://dx.doi.org/10.1109/CoASE.2012. 6386483

[23] N. Karmarkar, "A new polynomial-time algorithm for linear programming," in Proceedings of the sixteenth annual ACM symposium on Theory of computing. ACM, 1984, pp. 302311.

[24] H. R. Tiwary, "On the hardness of computing intersection, union and minkowski sum of polytopes," Discrete \& Computational Geometry, vol. 40, no. 3, pp. 469-479, 2008.

[25] K. Fukuda, T. M. Liebling, and C. Lütolf, "Extended convex hull," Computational Geometry, vol. 20, no. 1, pp. 13-23, 2001.

[26] M. Herceg, M. Kvasnica, C. Jones, and M. Morari, "MultiParametric Toolbox 3.0," in Proc. of the European Control Conference, Zürich, Switzerland, July 17-19 2013, pp. 502510 . 